\title{
Order-type Henstock and McShane integrals in Banach lattice setting
}

\author{
Domenico Candeloro* and Anna Rita Sambucini** \\ *University of Perugia-Dipartimento di Matematica e Informatica, via Vanvitelli, 1 I-06123 Perugia (Italy) \\ ** University of Perugia-Dipartimento di Matematica e Informatica, via Vanvitelli, 1 I-06123 Perugia (Italy) \\ E-mails:domenico.candeloro@unipg.it;anna.sambucini@unipg.it
}

\begin{abstract}
Henstock-type integrals are studied for functions defined in a compact metric space $T$ endowed with a regular $\sigma$-additive measure $\mu$, and taking values in a Banach lattice $X$. In particular, the space $[0,1]$ with the usual Lebesgue measure is considered.

The norm- and the order-type integral are compared and interesting results are obtained when $X$ is an $L$-space.
\end{abstract}

2010 AMS Mathematics Subject Classification: 28B20, $46 \mathrm{G} 10$.

Keywords: Banach space, Banach lattice, order-continuity, Henstock integral, McShane integral.

\section{INTRODUCTION}

It is well-known that many different notions of integral were introduced in the last century, for real-valued functions, in order to generalize the Riemann one. An exhaustive discussion of the various definitions for real-valued functions can be found in [31], where the Henstock-Kurzweil, the Lebesgue, and the McShane notions have been finely compared, and the equivalence between the McShane and the Lebesgue integrals is clearly described. The situation changes deeply in the case of Banach space-valued functions: in this case, it is wellknown that the stronger type of integral is the Bochner one, which implies the Birkhoff integrability, which in turn is stronger than the McShane and the latter is stronger than both Henstock and Pettis integrals. The wide literature in this topic witnesses the great interest for these problems: see for example [2], [9], [10], [8], [3], [11], [15], [24], [19], [18], [20], [17], [22], [23], [35], [37], [38], [36], [39]. Alternative notions of integrals have also been given, for various applications (see e.g. [27], [28], [29], [32]). Subsequently, the notions of order-type integrals have also been introduced and studied, for functions taking their values in ordered vector spaces, and in Banach lattices in particular: see [12], [25], [34], [6], [7], [1], [14], [13], [4]. Also the multivalued case has been intensively studied, for both types of convergences: see for example [16], [5].

In this research mainly the differences between norm- and order-type McShane integrals have been discussed, for singlevalued functions taking values in a Banach lattice with ordercontinuous norm.

However this note is just an anticipation of a forthcoming extended paper, with further results and more detailed proofs.
After a section of preliminaries, in the third one norm and order-type integrals are compared, showing a first striking difference: order integrals in general do not respect almost everywhere equality, except for order-bounded functions. Another interesting difference is that order integrals enjoy the socalled Henstock Lemma: this fact has interesting consequences in $L$-spaces, where McShane order integrability is stronger than the Bochner (norm) one. In the fourth section integrability in $[0,1]$ is discussed and it is proven that monotone mappings are $\mathrm{McShane}$ order-integrable, by using a similar procedure as in [31].

\section{PRELIMINARIES}

From now on, $T$ will denote a compact metric space, and $\mu: \mathscr{B} \rightarrow \mathbb{R}_{0}^{+}$any regular, nonatomic $\sigma$-additive measure on the $\sigma$-algebra $\mathscr{B}$ of Borel subsets of $T$.

A gage is any map $\gamma: T \rightarrow \mathbb{R}^{+}$. A partition $\Pi$ of $[0,1]$ is a finite family $\Pi=\left\{\left(E_{i}, t_{i}\right): i=1, \ldots, k\right\}$ of pairs such that the $E_{i}$ are pairwise disjoint sets whose union is $T$ and the points $t_{i}$ are called tags. If all tags satisfy the condition $t_{i} \in E_{i}$ then the partition is said to be of Henstock type, or a Henstock partition. Otherwise, it is said to be a free or McShane partition.

Given a gage $\gamma$, a partition $\Pi$ is $\gamma$-fine $(\Pi \prec \gamma)$ if $d\left(w, t_{i}\right)<\gamma\left(t_{i}\right)$ for every $w \in E_{i}$ and $i=1, \ldots, k$.

Clearly, a gage $\gamma$ can also be defined as a mapping associating with each point $t \in T$ an open ball centered at $t$ : sometimes this concept will be used, without risk of confusion.

Let $X$ be any Banach lattice with an order-continuous norm. For the sake of completeness the main notions of gauge integral are recalled here.

Definition 1: A function $f: T \rightarrow X$ is norm-integrable if there exists $J \in X$ such that, for every $\varepsilon>0$ there is a gage $\gamma: T \rightarrow \mathbb{R}^{+}$such that for every $\gamma$-fine Henstock partition of $T$, $\Pi=\left\{\left(E_{i}, t_{i}\right), i=1, \ldots, q\right\}$, it is:

$$
\|\sigma(f, \Pi)-J\| \leq \varepsilon
$$

(Here, as usual, the symbol $\sigma(f, \Pi)$ means $\left.\sum_{i=1}^{q} f\left(t_{i}\right) \mu\left(E_{i}\right)\right)$. In the case of integrability in the Henstock sense, this will be denoted with $\mathrm{H}$-integrability and the integral $J$ will be denoted with $H \int f d \mu$. 
Remark 2: It is worth noticing here that, in the previous definition, taking more generally free $\gamma$-fine partitions does not modify the concept introduced: in other words, the same integral is obtained if all free $\gamma$-fine partitions are allowed in the previous definition: see for example [5, Proposition 2.3]).

Parallel to this definition, notions of order-type integral can be given, in accordance with the following

Definition 3: A function $f: T \rightarrow X$ is order-integrable in the Henstock sense if there exist $J \in X$, an $(o)$-sequence $\left(b_{n}\right)_{n}$ in $X$ and a corresponding sequence $\left(\gamma_{n}\right)_{n}$ of gages, such that for every $n$ and every $\gamma_{n}$-fine Henstock partition of $T, \Pi=$ $\left\{\left(E_{i}, t_{i}\right), i=1, \ldots, q\right\}$, one has

$$
|\sigma(f, \Pi)-J| \leq b_{n} .
$$

In the case of integrability in the Henstock sense, this will be denoted with $(\mathrm{oH})$-integrability and the integral $J$ will be denoted with $(o H) \int f$.

It is obvious that also in this case there is no difference in taking all free $\gamma_{n}$-fine partitions in the previous definition. Thanks to the order continuity of the norm in $X$, it is easy to see that any $(\mathrm{oH})$-integrable map $f$ is also H-integrable and the integrals coincide. Furthermore, it can be observed that (oH)-integrability of a function $f: T \rightarrow X$ implies also Pettis integrability, thanks to well-known results concerning the McShane norm-integral: see [22, Theorem 8]. However, later it will be shown that the $(\mathrm{H})$ - and the $(\mathrm{oH})$-integrability are not equivalent, in general.

\section{COMPARISON BETWEen NORM AND ORDER INTEGRAL}

There are deep differences between order-type and normtype integrals. A first remarkable fact is that, in general, almost equal functions can behave in different ways with respect to the (oH)-integral, as it was proven in [1] Example 2.8], where a function $f:[0,1] \rightarrow c_{00}$ is given with the following properties: $f$ is almost everywhere null (with respect to the Lebesgue measure) and $f$ is not $(\mathrm{oH})$-integrable. So, this function is almost everywhere equal to 0 , hence is Bochner-integrable, but not $(\mathrm{oH})$-integrable or order-bounded. Indeed, for orderbounded functions, the situation is better, as shown in the next Proposition.

Proposition 4: Let $f, g: T \rightarrow X$ be two bounded maps, such that $f=g \mu$-almost everywhere. Then, $f$ is (oH)-integrable if and only if $g$ is, and the integral is the same.

Proof: Let $M$ be any majorant for $|f|$ and $|g|$, and assume that $f$ is (oH)-integrable, with integral $J$. Let $\left(b_{n}\right)_{n}$ and $\left(\gamma_{n}\right)_{n}$ be the sequences related to (oH)-integrability of $f$. In order to show integrability of $g$, fix $n$, and pick any open set $A_{n} \subset T$, with $\mu\left(A_{n}\right)<n^{-1}$ and $A_{n} \supset N:=\{t \in T: f(t) \neq g(t)\}$. Now, for each element $u \in N$ let $\delta_{n}(u)$ be any open set containing $u$ and contained in $A_{n}$ : then define $\gamma_{n}^{\prime}(t)=\gamma_{n}(t)$ when $t \notin N$, while $\gamma_{n}^{\prime}(u)=\gamma_{n}(u) \cap \delta_{n}(u)$ when $u \in N$.

Now, fix any tagged $\gamma_{n}^{\prime}$-fine partition $\Pi,\left(\Pi:=\left(E_{i}, \tau_{i}\right)_{i}\right)$ and observe that, whenever the tag $\tau_{i}$ belongs to $N$, then $E_{i} \subset A_{n}$.
So, it follows easily that

$$
\sup \left\{\sum_{\tau_{i} \in N}\left|f\left(\tau_{i}\right)\right| \mu\left(E_{i}\right), \sum_{\tau_{i} \in N}\left|g\left(\tau_{i}\right)\right| \mu\left(E_{i}\right)\right\} \leq \frac{M}{n},
$$

while

$$
\sum_{\tau_{i} \notin N} f\left(\tau_{i}\right) \mu\left(E_{i}\right)=\sum_{\tau_{i} \notin N} g\left(\tau_{i}\right) \mu\left(E_{i}\right),
$$

and finally

$$
\begin{aligned}
& |\sigma(g, \Pi)-J| \leq \\
& \leq|\sigma(f, \Pi)-J|+\sum_{\tau_{i} \in N}\left|f\left(\tau_{i}\right)\right| \mu\left(E_{i}\right)+ \\
& +\sum_{\tau_{i} \in N}\left|g\left(\tau_{i}\right)\right| \mu\left(E_{i}\right) \leq b_{n}+2 \frac{M}{n} .
\end{aligned}
$$

This clearly proves that $g$ is $(\mathrm{oH})$-integrable with integral $J$. Finally, interchanging the role of $f$ and $g$, the assertion follows.

Another interesting difference is in the validity of the so-called Henstock Lemma: indeed, $(\mathrm{oH})$-integrability yields this result, contrarily to the case of the norm-integral.

A Cauchy-type criterion is stated first in order to prove the existence of the $(\mathrm{oH})$-integral. The proof is straightforward.

Theorem 5: Let $f: T \rightarrow X$ be any mapping. Then $f$ is (oH)integrable if and only if there exist an $(o)$-sequence $\left(b_{n}\right)_{n}$ and a corresponding sequence $\left(\gamma_{n}\right)_{n}$ of gages, such that for every $n$, as soon as $\Pi, \Pi^{\prime}$ are two $\gamma_{n}$-fine Henstock partitions, the following holds:

$$
\left|\sigma(f, \Pi)-\sigma\left(f, \Pi^{\prime}\right)\right| \leq b_{n}
$$

Now, a Henstock-type lemma is stated, for the (oH)-integral. The proof is similar to that of [13, Theorem 1.4], and follows from the Cauchy criterion. However, some details are also given here.

Proposition 6: Let $f: T \rightarrow X$ be any (oH)-integrable function. Then, there exist an $(o)$-sequence $\left(b_{n}\right)_{n}$ and a corresponding sequence $\left(\gamma_{n}\right)_{n}$ of gages, such that, for every $n$ and every $\gamma_{n}$-fine Henstock partition $\Pi$ it is

$$
\sum_{E \in \Pi} O b_{n}(f, E) \leq b_{n}
$$

where

$$
O b_{n}(E)=\sup _{\Pi_{E}^{\prime}, \Pi_{E}^{\prime \prime}}\left\{\left|\sum_{F^{\prime \prime} \in \Pi_{E}^{\prime \prime}} f\left(\tau_{F^{\prime \prime}}\right) \mu\left(F^{\prime \prime}\right)-\sum_{F^{\prime} \in \Pi_{E}^{\prime}} f\left(\tau_{F^{\prime}}\right) \mu\left(F^{\prime}\right)\right|\right\},
$$

and $\Pi_{E}^{\prime}, \Pi_{E}^{\prime \prime}$ run along all $\gamma_{n}$-fine Henstock partitions of $E$.

Proof: First observe that, thanks to the Cauchy criterion, an $(o)$-sequence $\left(b_{n}\right)_{n}$ exists, together with a corresponding sequence of gages $\left(\gamma_{n}\right)_{n}$, such that

$$
\left|\sum_{F^{\prime} \in \Pi^{\prime}} f\left(\tau_{F^{\prime}}\right) \mu\left(F^{\prime}\right)-\sum_{F^{\prime \prime} \in \Pi^{\prime \prime}} f\left(\tau_{F^{\prime \prime}}\right) \mu\left(F^{\prime \prime}\right)\right| \leq b_{n}
$$


(with obvious meaning of symbols) holds, for all $\gamma_{n}$-fine partitions $\Pi^{\prime}, \Pi^{\prime \prime}$. Now, take any $\gamma_{n}$-fine partition $\Pi$, and, for each element $E$ of $\Pi$, consider two arbitrary subpartitions $\Pi_{E}^{\prime}$ and $\Pi_{E}^{\prime \prime}$. Then, taking the union of the subpartitions $\Pi_{E}^{\prime}$ as $E$ varies, and making the same operation with the subpartitions $\Pi_{E}^{\prime \prime}$, two $\gamma_{n}$-fine partitions of $T$ are obtained, for which (2) holds true. From (2), obviously it follows

$$
\sum_{F^{\prime} \in \Pi^{\prime}} f\left(\tau_{F^{\prime}}\right) \mu\left(F^{\prime}\right)-\sum_{F^{\prime \prime} \in \Pi^{\prime \prime}} f\left(\tau_{F^{\prime \prime}}\right) \mu\left(F^{\prime \prime}\right) \leq b_{n} .
$$

Now, let $E_{1}$ be the first element of $\Pi$. In the summation at lefthand side, fix all the $F^{\prime} s$ and the $F^{\prime \prime} s$ that are not contained in $E_{1}$. Taking the supremum when the remaining $F^{\prime} s$ and $F^{\prime \prime} s$ vary in all possible ways, it follows

$$
O b_{n}\left(f, E_{1}\right)+\sum_{\substack{F^{\prime} \in \Pi^{\prime}, F^{\prime} \not \subset E_{1}}} f\left(\tau_{F^{\prime}}\right) \mu\left(F^{\prime}\right)-\sum_{\substack{F^{\prime \prime} \in \Pi^{\prime \prime}, F^{\prime \prime} \not \subset E_{1}}} f\left(\tau_{F^{\prime \prime}}\right) \mu\left(F^{\prime \prime}\right) \leq b_{n} .
$$

In the same fashion, fixed all the $F^{\prime}$ and $F^{\prime \prime}$ that are not contained in the second subset of $\Pi$, (say $E_{2}$ ), and making the same operation, it follows

$$
\begin{aligned}
& O b_{n}\left(f, E_{1}\right)+O b_{n}\left(f, E_{2}\right)+\sum_{\substack{F^{\prime} \in \Pi^{\prime}, F^{\prime} \not \subset E_{1} \cup E_{2}}} f\left(\tau_{F^{\prime}}\right) \mu\left(F^{\prime}\right)+ \\
& -\sum_{\substack{F^{\prime \prime} \in \Pi^{\prime \prime}, F^{\prime \prime} \not \subset E_{1} \cup E_{2}}} f\left(\tau_{F^{\prime \prime}}\right) \mu\left(F^{\prime \prime}\right) \leq b_{n}
\end{aligned}
$$

Now, it is clear how to deduce the assertion.

Remark 7: A first consequence of the previous Proposition 6 is that any $(\mathrm{oH})$-integrable function $f$ is also integrable in the same sense in every measurable subset $A$. Indeed, taking the same $(o)$-sequence $\left(b_{n}\right)_{n}$ and the same corresponding sequence $\left(\gamma_{n}\right)_{n}$ as for integrability of $f$, for each $n$ any $\gamma_{n}$-fine partition of $A$ can be extended to a $\gamma_{n}$-fine partition of $T$ thanks to the Cousin Lemma, and so, for any two $\gamma_{n}$-fine partitions $\Pi, \Pi^{\prime}$ of $A$, it follows

$$
\left|\sigma(f, \Pi)-\sigma\left(f, \Pi^{\prime}\right)\right| \leq O b_{n}(f, A) \leq b_{n} .
$$

Then, the Cauchy criterion yields the conclusion.

Remark 8: By means of usual techniques, one also proves additivity of the integral: namely whenever $f$ is integrable in $T$, and $A, B$ are two disjoint measurable subsets of $T$, then $\int f 1_{A \cup B} d \mu=\int_{A} f d \mu+\int_{B} f d \mu$.

The following Theorem collects some easy consequences of Proposition 6.

Theorem 9: Let $f: T \rightarrow X$ be any (oH)-integrable function. Then there exist an $(o)$-sequence $\left(b_{n}\right)_{n}$ and a corresponding sequence $\left(\gamma_{n}\right)_{n}$ of gages, such that:

9.1) for every $n$ and every $\gamma_{n}$-fine partition $\Pi$ one has

$$
\sum_{E \in \Pi}\left|f\left(\tau_{E}\right) \mu(E)-(\mathrm{oH}) \int_{E} f d \mu\right| \leq b_{n}
$$

9.2) for every $n$ and every $\gamma_{n}$-fine partition $\Pi$ it holds

$$
\sum_{E \in \Pi}\left|f\left(\tau_{E}\right) \mu(E)-f\left(\tau_{E}^{\prime}\right) \mu(E)\right| \leq b_{n},
$$

as soon as all the tags satisfy the condition $E \subset$ $\gamma_{n}\left(\tau_{E}^{\prime}\right)$ and $E \subset \gamma_{n}\left(\tau_{E}\right)$ for all $E$.

Remark 10: For further reference, observe that in the theorem above all partitions may also be free, since, as already noticed, the restriction $\tau_{E} \in E$ does not affect the results.

A consequence of this theorem is that the $(\mathrm{oH})$-integrability of $f$ implies the $(\mathrm{oH})$-integrability of $|f|$.

Theorem 11: If $f: T \rightarrow X$ is (oH)-integrable, then also $|f|$ is.

Proof: The Cauchy criterion is used in the following formulation: there exist an $(o)$-sequence $\left(b_{n}\right)_{n}$ and a corresponding sequence $\left(\gamma_{n}\right)_{n}$ of gages, such that, for each $n$, as soon as $\Pi, \Pi^{\prime}$ are $\gamma_{n}$-fine free partitions and $\Pi^{\prime}$ is finer than $\Pi$,

$$
\left|\sum_{E \in \Pi}\right| f\left(\tau_{E}\right)\left|\mu(E)-\sum_{E^{\prime} \in \Pi^{\prime}}\right| f\left(\tau_{E^{\prime}}\right)\left|\mu\left(E^{\prime}\right)\right| \leq b_{n} .
$$

Now, if $\left(b_{n}\right)_{n}$ and $\left(\gamma_{n}\right)_{n}$ are as in Theorem 9 and $\Pi$ and $\Pi^{\prime}$ are as above, it is

$$
\begin{aligned}
& \sum_{E \in \Pi}\left|f\left(\tau_{E}\right)\right| \mu(E)-\sum_{E^{\prime} \in \Pi^{\prime}}\left|f\left(\tau_{E^{\prime}}\right)\right| \mu\left(E^{\prime}\right)= \\
= & \sum_{E \in \Pi} \sum_{E^{\prime} \in \Pi^{\prime},}\left(\left|f\left(\tau_{E}\right)\right|-\left|f\left(\tau_{E^{\prime}}\right)\right|\right) \mu\left(E^{\prime}\right)= \\
= & \sum_{E^{\prime} \in \Pi^{\prime}}\left(\left|f\left(\tau_{E^{\prime}}^{*}\right)\right|-\left|f\left(\tau_{E^{\prime}}\right)\right|\right) \mu\left(E^{\prime}\right),
\end{aligned}
$$

where the tags $\tau_{E^{\prime}}^{*}$ coincide with $\tau_{E}$ whenever $E^{\prime} \subset E, E \in \Pi$. Therefore, a simple application of 92) (and Remark 10) leads to (4) and the proof is finished.

The last theorem can be compared with a previous result by Drewnowski and Wnuk, ([21, Theorem 1]), where the Bochner integral is considered, for functions taking values in a Banach lattice $X$, and it is proven that the modulus of the indefinite Bochner integral of $f$ is precisely the indefinite integral of $|f|$.

Here a similar result for $(\mathrm{oH})$-integrable mappings is stated, after introducing a new definition.

Definition 12: Let $f: T \rightarrow X$ be any (oH)-integrable mapping, and set

$$
\mu_{f}(A)=(\mathrm{oH}) \int_{A} f d \mu
$$

for all Borel sets $A \in \mathscr{B}$. Then $\mu_{f}$ is said to be the indefinite integral of $f$. The modulus of $\mu_{f}$, denoted by $\left|\mu_{f}\right|$, is defined for each $A \in \mathscr{B}$ as follows:

$$
\left|\mu_{f}\right|(A)=\sup \left\{\sum_{B \in \pi}\left|\mu_{f}(B)\right|: \pi \in \Pi(A)\right\}
$$

where $\Pi(A)$ is the family of all finite partitions of $A$. (The boundedness of this quantity will be proven soon).

Now the following theorem can be stated. 
Theorem 13: Assume that $f: T \rightarrow X$ is (oH)-integrable. Then one has

$$
\left|\mu_{f}\right|=\mu_{|f|}
$$

Proof: First of all, observe that $|f|$ is integrable too, thanks to Theorem 11 Since clearly

$$
\left|(\mathrm{oH}) \int_{B} f d \mu\right| \leq(\mathrm{oH}) \int_{B}|f| d \mu
$$

holds for every set $B \in \mathscr{A}$, it follows that

$$
\left|\mu_{f}\right| \leq \mu_{|f|}
$$

This also shows that the modulus $\left|\mu_{f}\right|$ is bounded. Now, since $\left|\mu_{f}\right|$ and $\mu_{|f|}$ are additive, in order to obtain the reverse inequality, it will be sufficient to prove that $\mu_{|f|}(T)=\left|\mu_{f}\right|(T)$. To this aim, the Henstock Lemma, 9, will be used, and in particular 91). Let $\left(b_{n}\right)_{n}$ and $\left(\gamma_{n}\right)_{n}$ be an $(o)$-sequence and its corresponding sequence of gages, related to integrability of both $f$ and $|f|$. So, for every $n$ and every $\gamma_{n}$-fine partition $\pi \equiv\left(E_{i}, t_{i}\right)_{i}$ it holds

$$
\begin{aligned}
\mu_{|f|}(T) \quad-\left|\mu_{f}\right|(T) \leq \sum_{i}\left(\mu_{|f|}\left(E_{i}\right)-\left|(\mathrm{oH}) \int_{E_{i}} f d \mu\right|\right) \leq \\
\leq \quad \sum_{i}\left(\mu_{|f|}\left(E_{i}\right)-\left|f\left(t_{i}\right)\right| \mu\left(E_{i}\right)\right)+ \\
+\quad \sum_{i}\left(\left|f\left(t_{i}\right)\right| \mu\left(E_{i}\right)-\left|(\mathrm{oH}) \int_{E_{i}} f d \mu\right|\right) \leq 2 b_{n}
\end{aligned}
$$

Since $\left(b_{n}\right)_{n}$ is an $(o)$-sequence, then $\mu_{|f|}(T) \leq\left|\mu_{f}\right|(T)$, and so clearly also $\mu_{|f|}(T)=\left|\mu_{f}\right|(T)$. This concludes the proof.

Another interesting consequence is concerned with $L$ spaces. Recall that a Banach lattice $X$ is an $L$-space if its norm $\|\cdot\|$ satisfies

$$
\|x+y\|=\|x\|+\|y\|
$$

for all positive elements $x, y$ in $X$ (see also [25]).

The following definition, related with norm-integrability, is needed.

Definition 14: [18, Definition 3] $f: T \rightarrow X$ is variationally $\mathrm{H}$ integrable (in short $\mathrm{vH}$-integrable) if for every $\varepsilon>0$ there exists a gage $\gamma$ such that, for every $\gamma$-fine partition $\Pi \equiv\left(E, t_{E}\right)_{E}$ the following holds:

$$
\sum_{E \in \Pi}\left\|f\left(t_{E}\right) \mu(E)-(\mathrm{H}) \int_{E} f d \mu\right\| \leq \varepsilon .
$$

For results on this setting see also [18].

Theorem 15: Let $f: T \rightarrow X$ be $(\mathrm{oH})$-integrable, and assume that $X$ is an $L$-space. Then $f$ is Bochner integrable.

Proof: In order to prove the Bochner integrability, it will suffice to show that $f$ is vH-integrable. Indeed, by [19. Theorem 2], the variational integrability implies the Bochner integrability. The H-integrability of $\|f\|$ will be proved first. In accordance with the previous Theorem 9 and with the same meanings of symbols, there exist an $(o)$-sequence $\left(b_{n}\right)_{n}$ and a corresponding sequence $\left(\gamma_{n}\right)_{n}$ of gages, such that, for every $n$ and every $\gamma_{n}$-fine partition $\Pi$ one has

$$
\sum_{E \in \Pi}\left|f\left(\tau_{E}\right) \mu(E)-f\left(\tau_{E}^{\prime}\right) \mu(E)\right| \leq b_{n} .
$$

Since the norm of $X$ is compatible with the order, then $\lim _{n}\left\|b_{n}\right\|=0$. So, fix $\varepsilon>0$ and pick any integer $N$ such that $\left\|b_{N}\right\| \leq \varepsilon$. Then, if $\Pi$ is any $\gamma_{N}$-fine partition, we have

$$
\left\|\sum_{E \in \Pi}\left|f\left(\tau_{E}\right) \mu(E)-f\left(\tau_{E}^{\prime}\right) \mu(E)\right|\right\| \leq\left\|b_{N}\right\| \leq \varepsilon,
$$

in accordance with 92 ). Thanks to the particular nature of the norm $\|\cdot\|$, we deduce that

$$
\sum_{E \in \Pi}\left\|\left|f\left(\tau_{E}\right) \mu(E)-f\left(\tau_{E}^{\prime}\right) \mu(E)\right|\right\| \leq\left\|b_{N}\right\| \leq \varepsilon,
$$

and so

$$
\sum_{E \in \Pi}\left|\left\|f\left(\tau_{E}\right)\right\|-\left\|f\left(\tau_{E}^{\prime}\right)\right\|\right| \mu(E) \leq\left\|b_{N}\right\| \leq \varepsilon,
$$

as soon as $\Pi$ is $\gamma_{N}$-fine, both for the tags $\tau_{E}$ and for the tags $\tau_{E}^{\prime}$.

Now, proceeding as in the proof of Theorem 11 it is not difficult to prove that $\|f\|$ satisfies the Cauchy criterion for the Henstock integrability, and therefore it is integrable. From (6), also thanks to 91), it is easy to deduce also (5). In conclusion, $f$ is variationally integrable, its norm is integrable, and then, from Pettis integrability, it follows also the Bochner integrability.

Remark 16: The previous result can be used to show that the $(\mathrm{H})$-integrability in general does not imply the $(\mathrm{oH})$ integrability: indeed, if $X$ is any infinite-dimensional Banach space, there exists a McShane (norm)-integrable map $f:[0,1] \rightarrow X$ that is not Bochner integrable (see [39]). In particular, when $X$ is an $L$-space (of infinite dimension), such function $f$ cannot be $(\mathrm{oH})$-integrable, in view of Theorem 15 .

\section{INTEGRABILITY IN $[0,1]$}

In this section the $(\mathrm{oH})$-integrability of functions $f:[0,1] \rightarrow X$ is studied, where $[0,1]$ is endowed with the usual Lebesgue measure $\lambda$.

From now on, only free partitions consisting of subintervals of $[0,1]$ are considered, rather than arbitrary measurable subsets. Indeed, in [24] it is proven that there is equivalence between the two types: though the proof there is related only to norm integrals, the technique is the same. For this reason, from now on the symbol (oM)-integral will be used rather than $(\mathrm{oH})$-integral.

A useful result, parallel to [31, Lemma 5.35], is the following:

Lemma 17: Let $f:[0,1] \rightarrow X$ be any fixed function, and suppose that there exists an $(o)$-sequence $\left(b_{n}\right)_{n}$ such that, for 
every $n$ two (oM)-integrable functions $g_{1}$ and $g_{2}$ can be found, with the same regulating $(o)$-sequence $\left(\beta_{n}\right)_{n}$, such that $g_{1} \leq$ $f \leq g_{2}$ and $(\mathrm{oM}) \int g_{2} d \lambda \leq(\mathrm{oM}) \int g_{1} d \lambda+b_{n}$. Then $f$ is (oM)integrable.

The fact that increasing functions are (oM)-integrable can be deduced similarly as in [31, example 5.36].

Theorem 18: Let $f:[0,1] \rightarrow X$ be increasing. Then $f$ is (oM)-integrable.

Remark 19: In a similar way, one can prove the (oM)integrability more generally for (order bounded) mappings that are Riemann-integrable in the order sense.

\section{CONCLUSiON}

In this paper the notions of Henstock and McShane integrability for functions defined in a metric compact regular space and taking values in a Banach lattice with an ordercontinuous norm are investigated. Both the norm-type and the order-type integrals have been studied and compared. Though in general the order-type integral is stronger than the normone, in $M$-spaces the two notions coincide, while in $L$-spaces the order-type Henstock integral is indeed a Bochner one. Finally, the particular case of functions defined in a real interval is considered, and it is proven that monotone mappings are always order-McShane integrable.

Acknowledgment The authors have been supported by University of Perugia - Department of Mathematics and Computer Sciences - Grant Nr 2010.011.0403, Prin "Metodi logici per il trattamento dell'informazione", Prin "Descartes" and by the Grant prot. U2014/000237 of GNAMPA - INDAM (Italy).

\section{REFERENCES}

[1] A. Boccuto - A.M. Minotti - A.R. Sambucini, Set-valued KurzweilHenstock integral in Riesz space setting, PanAmerican Mathematical Journal 23 (1) (2013), 57-74.

[2] A. Boccuto, A. V. Bukhvalov, A. R. Sambucini Inequalities in Classical Spaces with Mixed Norms, Positivity 6, (2002) 393-411.

[3] A. Boccuto - D. Candeloro - A. R. Sambucini A Fubini Theorem in Riesz Spaces for the Kurzweil-Henstock Integral, Journal of Function Spaces and Applications, 9, No. 3 (2011), 283-304.

[4] A. Boccuto, D. Candeloro, A.R. Sambucini Vitali-type theorems for filter convergence related to vector lattice-valued modulars and applications to stochastic processes J. Math. Anal. Appl. 419 (2), (2014), 818-838; doi: 10.1016/j.jmaa-2014.05.014

[5] A. Boccuto, D. Candeloro, A. R. Sambucini A note on set-valued Henstock-McShane integral in Banach (lattice) space setting, (2014), submitted, arXiv:1405.6530 [math.FA].

[6] A. Boccuto, X. Dimitriou, Nikodym theorems for lattice group-valued measures with respect to filter convergence, Adv. Pure Math. 4 (5) (2014), 213-221.

[7] A. Boccuto, X. Dimitriou, N. Papanastassiou, Schur lemma and limit theorems in lattice groups with respect to filters, Math. Slovaca 62 (6) (2012), 1145-1166.

[8] A. Boccuto, A. R. Sambucini The Henstock-Kurzweil integral for functions defined on unbounded intervals and with values in Banach spaces Acta Mathematica (NITRA) 7, (2004) 3-17.

[9] A. Boccuto, A. R. Sambucini, A McShane Integral for Multifunctions, J. Concr. Appl. Math. 2 (4) (2004), 307-325.

[10] A. Boccuto, A. R. Sambucini, A note on comparison between Birkhoff and McShane-type integrals for multifunctions, Real Anal. Exchange 37 (2) (2012), 315-324.
[11] A. V. Bukhvalov, A. I. Veksler, V. A. Geiler, Normed lattices, Journal of Soviet Mathematics (1982) 18 (4), 516-551.

[12] A. V. Bukhvalov, A. I. Veksler, G. Ya Lozanovskii, Banach Lattices Some Banach Aspects of Their Theory, Russian Mathematical Surveys (1979), 34 (2),159-212. doi:10.1070/RM1979v034n02ABEH002909

[13] D. Candeloro, Riemann-Stieltjes integration in Riesz Spaces, Rend. Mat. Roma (Ser. VII), 16 (2) (1996), 563-585.

[14] D. Candeloro, A.R. Sambucini Filter convergence and decompositions for vector lattice-valued measures, in press in Mediterranean J. Math. doi: 10.1007/s00009-014-0431-0 arXiv:1401.7818 [math.FA].

[15] S. Cao The Henstock integral for Banach-valued functions, SEA Bull. Math. 16 (1992), 35-40.

[16] B. Cascales, V. Kadets, J. Rodríguez, The Pettis integral for multi-valued functions via single-valued ones J. Math. Anal. Appl. 332 (2007), no. $1,1-10$.

[17] R. Deville, J. Rodríguez, Integration in Hilbert generated Banach spaces, Israel J. Math. 177 (2010), 285-306.

[18] L. Di Piazza, V. Marraffa, The McShane, PU and Henstock integrals of Banach valued functions, Czechoslovak Math. Journal 52 (2002), 609633.

[19] L. Di Piazza, K. Musiał, A characterization of variationally McShane integrable Banach-space valued functions, Ill. J. Math. 45 (1) (2001), 279-289.

[20] L. Di Piazza, D. Preiss, When do McShane and Pettis integrals coincide?, Illinois Journal of Mathematics, 47 (4) (2003), 1177-1187.

[21] L. Drewnowski, W. Wnuk, On the modulus of indefinite vector integrals with values in Banach lattices, Atti Sem. Mat. Fis. Univ. Modena, 47 (1999), 221-233.

[22] D. H. Fremlin, The Henstock and McShane integrals of vector-valued functions Illinois J. Math. 38 (3) (1994), 471-479.

[23] D. H. Fremlin, J. Mendoza, On the integration of vector-valued functions Illinois J. Math. 38 (1) (1994), no. 1, 127-147.

[24] D. H. Fremlin, The generalized McShane integral Illinois J. Math. 39 (1) (1995), 39-67.

[25] D. H. Fremlin, Measure theory. Vol. 3. Measure Algebras, Torres Fremlin, Colchester, 2002.

[26] D. H. Fremlin, Measure theory. Vol. 4. Topological measure spaces, Torres Fremlin, Colchester, 2006.

[27] M. C. Isidori, A. Martellotti, A. R. Sambucini The Monotone integral, Atti Sem. Mat. Fis. Univ. Modena, suppl. Vol XLVI (1998), 803-825.

[28] M. C. Isidori, A. Martellotti, A. R. Sambucini The Bochner and the monotone integrals with respect to a nuclear finitely additive measure, Matematica Slovaca, 48, N. 4 (1998), 377-390.

[29] M. C. Isidori, A. R. Sambucini The monotone integral-part II, Atti Sem. Mat. Fis. Univ Modena, XLVI, (1998), 469-478.

[30] S. Kakutani, Concrete representation of abstract $(L)$-spaces and the mean ergodic theorem, Annals of Mathematics 42 (1941), 523-537.

[31] D.S. Kurtz, C.W. Swartz, Theories of Integration, the Integrals of Riemann, Lebesgue, Henstock-Kurzweil, and McShane, Series in Real Analysis Vol. 9, World Scientific (2004).

[32] C. C. A. Labuschagne, B. A. Watson, Discrete stochastic integration in Riesz spaces, Positivity 14 (4) (2010), 859-875.

[33] L. P. Lee, R. Výborný, The integral: An easy approach after Kurzweil and Henstock, (2000), Cambridge Univ. Press., Cambridge.

[34] P. Meyer-Nieberg, Banach lattices, (1991), Springer-Verlag, BerlinHeidelberg.

[35] B. Riečan, On the Kurzweil Integral in Compact Topological Spaces, Radovi Mat. 2 (1986), 151-163.

[36] J. Rodríguez, On the existence of Pettis integrable functions which are not Birkhoff integrable, Proc. Amer. Math. Soc. 133 (2005), no. 4, $1157-$ 1163.

[37] J. Rodríguez, On the equivalence of McShane and Pettis integrability in non-separable Banach spaces, J. Math. Anal. Appl. 350 (2009) 514-524.

[38] J. Rodríguez, Some examples in vector integration, Bull. of the Australian Math. Soc. (2009) 80 (03), 384 - 392.

[39] V.A. Skvortsov, A.P. Solodov, A variational integral for Banach-valued functions, Real Anal. Exchange 24 (1998-1999), 799-806. 